\title{
Etudes paleoenvironnementales dans la grotte «El Castillo» (Puente Viesgo, Cantabrie, Espagne)
}

\author{
Abdellah Dari ${ }^{*}$ et Josette Renault-Miskovsky*
}

\begin{abstract}
RESUMEN
ABSTRACT

Se presentan los datos obtenidos en

We present here some results related una serie de análisis polínicos with palinological analysis performed in puntuales realizados en los niveles the stratigraphical sequence of El musterienses y del auriñaciense de transición de la Cueva del Castillo. Los datos permiten situar estos niveles dentro de la dinámica de la transición Paleolítico Medio-Paleolítico Superior. Los datos se complementan con los resultados previos del análisis faunistico realizado sobre los materiales procedentes de las nuevas excavaciones

PALABRAS CLAVE: Palinología, Fauna, Transición Paleolítico Medio-Superior, El Castillo, Musteriense, Auriñaciense. Castillo cave. We present the results of the Mousterian and Transitional Aurignacian which place these units into the general framework of the transition between the Middle to Upper Paleolithic. In addition we compare the botanical data with the preliminary results of the faunal analysis.

KEY WORDS:

Palinology, Fauna, Middle-Upper paleolithic transition, El Castillo, Mousterian, Aurignacian.
\end{abstract}

* Abdellah Dari, Docteur. Josette Renault-Miskovsky, Directeur de Recherche au CNRS, Laboratoire de Préhistoire du Muséum National d'Histoire Naturelle, Institut de Paléontologie humaine, 1 rue René Panhard, 75013 Paris, France. Tél.: 0143316291 - Fax: 0143312279. 


\section{INTRODUCTION}

Lors d'une réunion de travail du G.D.R. "Analyse comparative de comportements symboliques et techniques à la fin du Paléolithique moyen et au Paléolithique supérieur en Europe", dirigé par Denis Vialou, l'un des groupes de réflexion sur "les matières premières animales - ivoire/bois de renne" a donné l'occasion de présenter quelques synthèses fournies par l'analyse de matériels de certains sites de référence dont El Castillo.

Nous avons alors constaté que les analyses polliniques tentées sur le site n'avaient pas encore abouti et nous avons proposé de faire quelques nouvelles investigations, dans l'Unité de Palynologie de l'Institut de Paléontologie humaine, en appliquant une méthode de lévigation en liqueur dense (chlorure de zinc) du dernier culot de centrifugation, après le traitement du sédiment par la méthode chimique classique ; ce procédé permet de recueillir la quintessence du matériel sporo-pollinique d'un sédiment, quand il est peu abondant.

Les résultats de la palynologie comparés aux observations réalisées sur le matériel osseux de la faune seront de la plus haute importance pour reconstituer l'environnement du site aux temps préhistoriques et les comportements de chasse de ses habitants.

Il faut en effet rappeler l'importance de la séquence stratigraphique et archéologique de la grotte du Castillo, l'une des plus vastes actuellement connu€s dans la péninsule ibérique (Cabrera Valdès et al., 1993). Elle s'ouvre à 195 mètres d'altitude dans les Cantabres, au Nord de l'Espagne, près de la ville de Santander (fig. 1) et présente une succession complète d'occupations paléolithiques, du Paléolithique inférieur au Paléolithique supérieur, avec la transition Paléolithique moyen - Paléolithique supérieur entre des niveaux moustériens (datations ESR, couches 22 et 21 : moyenne de 70 - 69000 ans ; Rink et al., 1997 - datations $\mathrm{C}_{14}$, couche 20. 40000 ans ; Cabrera Valdès, 1996) et l'Aurignacien de Transition, dont les premières manifestations se placeraient aussi il y a environ 40000 ans (datations $\mathrm{C}_{14}$; Cabrera Valdès et al., 1996), c'est-à-dire quelques millénaires plus tôt dans le Nord de l'Espagne que dans les autres parties de l'Europe de l'ouest.

\section{ETUDE PALYNOLOGIQUE PRELIMINAIRE}

Six échantillons appartenant à des niveaux culturels différents, nous ont été confiés par V. Cabrera Valdès et F. Bernaldo de Quiros en vue d'une étude pollinique préliminaire. Ce sont : 


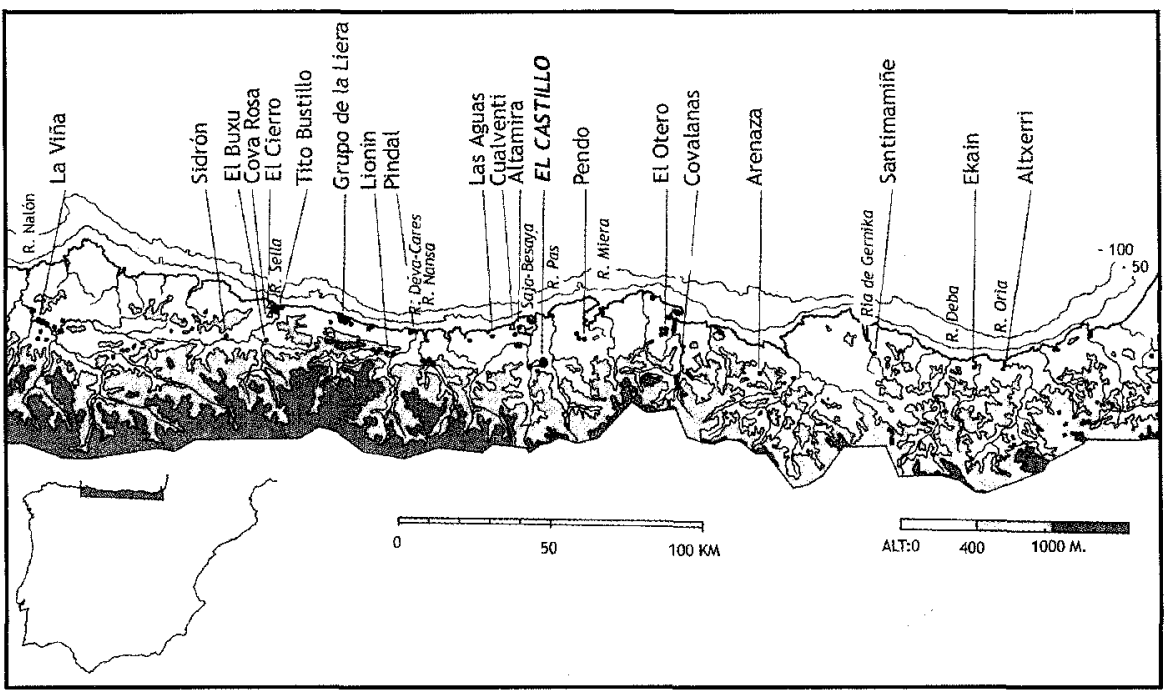

Fig. 1. Situation géogrphique de la grotte El Castillo (Puente Viesgo, Cantabrie, Espagne), d'après Moure Romanillo, 1994.

- échantillon 1 - niveau 24 - Acheuléen

- échantillon 2 - niveau 22 - Moustérien Bêta

- échantillon 3 - niveau 21 - Moustérien entre Bêta et Alpha

- échantillon 4 - niveau 20 - Moustérien Alpha

- échantillon 5 - niveau 18C - Aurignacien de Transition

- échantillon 6 - niveau 18B - Aurignacien de Transition

Les résultats ont été présentés (fig. 2) sous forme d'histogrammes synthétiques qui figurent pour chaque niveau et de la gauche vers la droite:

L'ensemble des taxons rencontrés, groupés par affinités écologiques à savoir, le Pin, les taxons tempérés et thermophiles (Alnus, Betula, Carpinus, Corylus, Hedera, Juglans, Quercus t. pedunculata), les taxons méditerranéens (Cistus, Quercus t. ilex), les ligneux et herbacées xérophiles et/ou steppiques (Cupressaceae, Ephedra, Asteraceae t. échinulé, Artemisia, Centaurea, Asteraceae t. fenestré, Caryophyllaceae, Poaceae, Thalictrum), les herbacées ubiquistes (Apiaceae, Brassicaceae, Convolvulaceae, Cyperaceae, Ericaceae, Fabaceae, Lamiaceae, Liliaceae, Plantago, une Potamogetonaceae isolée, Ranunculaceae, Rubiaceae, Scrofulariaceae, Urticaceae). 

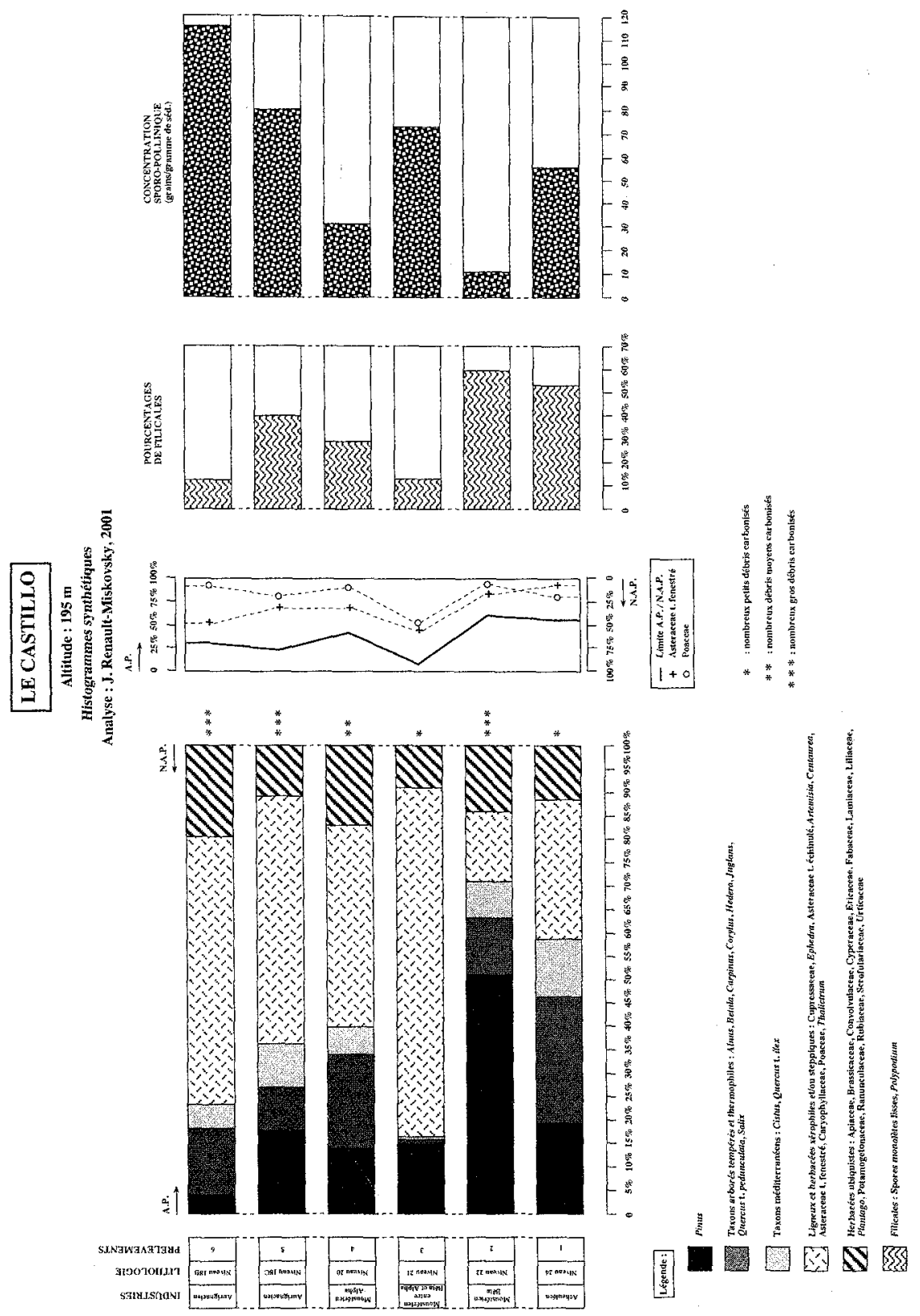

Fig. 2. Palynologie à la grotte du Castillo; histogrammes synthétiques. 
- La courbe AP - NAP

t. fenestré

- Les courbes des deux principales herbacées: Poaceae et Asteraceae

- Les Filicales (spores monolètes lisses et Polypodium)

- La valeur de la concentration pollinique.

\section{Remarques}

1. Les niveaux $24,22,20$ et $18 \mathrm{~B}$, ont livré quelques grains de pollen d'une Myrtacée que nous avons prudemment écartée de nos comptages ; les grains sont bien conservés et nous savons qu'une plantation d'Eucalyptus globulus occupe une partie de la végétation actuelle près du site (Uzquiano y Cabrera V., 1999).

2. La richesse pollinique est dans l'absolu toujours faible : entre 10 grains et 115 grains par gramme de sédiments ; elle varie cependant d'un niveau à l'autre et notre appréciation est toute relative.

Ainsi nous pouvons reconstituer l'environnement végétal contemporain de chacune des périodes culturelles envisagées:

Remarque : la lecture d'une lame par niveau a fourni une moyenne de 200 grains et de 20 taxons sauf exception.

1 - niveau 24 - Acheuléen

- Richesse pollinique moyenne - 26 taxons.

- AP est voisin de $60 \%$

- Les arbres représentés sont : le Pin, les taxons tempérés et thermophiles et quelques taxons méditerranéens.

- Les genres et les familles xérophiles et/ou steppiques atteignent $30 \%$.

- Le niveau 24 est le seul à enregistrer un taux de Poaceae supérieur à celui des Asteraceae de type fenestré.

- Le taux des Filicales dépasse $50 \%$.

Le climat contemporain de ce niveau acheuléen est semble-t-il tempéré chaud et assez sec ; il pourrait s'inscrire dans un épisode du stade isotopique 7 (ou 9?). 
2 - Niveau 22 - Moustérien Bêta

- Datation ESR $\approx 70000$ ans (Rink et al., 1997).

- Richesse pollinique très faible - 14 taxons.

- AP atteint plus de $70 \%$

- C'est le taux élevé (voisin de $50 \%$ ) de pollen de Pin qui donne sa forte valeur à AP.

- Le reste du couvert arboré est partagé entre les taxons tempérés et thermophiles et quelques taxons méditerranéens.

- Les genres et les familles xérophiles et/ou steppiques ne dépassent pas $15 \%$.

- A ce niveau, les Asteraceae de type fenestré prennent le pas sur les Poaceae.

- Le taux des Filicales atteint presque $60 \%$.

Le climat contemporain de ce dépôt moustérien est semble-t-il moins chaud et plus humide que le précédent.

Compte tenu du contexte culturel et de la datation ESR, nous sommes tentés de le placer à la limite supérieure du stade isotopique 5.

3 - Niveau 21 - Moustérien entre Bêta et Alpha

- Datation ESR $\approx 69000$ ans (Rink et al., 1997)

- Richesse pollinique moyenne - 17 taxons

- AP dépasse à peine $15 \%$

- C'est le Pin qui donne sa valeur à AP; quelques rares Cupressaceae et Chênes (un de type ilex et un de type pedunculata) l'accompagnent.

- Le paysage est presque entièrement occupé par les xérophiles et/ou steppiques.

- Les Asteraceae, en progression, dominent toujours les Poaceae.

- Le taux des Filicales n'atteint pas $15 \%$.

Le climat froid et sec contemporain de ce dépôt moustérien daté de 69 000 ans appartient vraisemblablement au stade isotopique 4 là la limite fin 5 - début 4). 
4 - Niveau 20 - Moustérien Alpha

- Datation $\mathrm{C}_{14} \approx 43-45000$ ans (Cabrera Valdès et al., 1996)

- Richesse pollinique faible - 20 taxons

- AP est voisin de $40 \%$.

- Le couvert arboré est partagé entre les Pins, les taxons tempérés et thermophiles et quelques genres méditerranéens.

- Les xérophiles et/ou steppiques sont encore nombreux ( $\approx 45 \%$ )

- Les Asteraceae supplantent encore les Poaceae.

- Le taux des Filicales est voisin de $30 \%$.

Le climat contemporain de ce niveau moustérien daté de 43-45 000 ans appartient à un épisode tempéré-froid relativement sec du stade isotopique 3.

La sédimentologie (Hoyos Gomez, in: Cabrera Valdès et al., 1993) révèle «un coup de froid de puissance moyenne à forte plus important que celui du niveau $18 »$.

Le niveau 19 est stérile

5 - Niveau 18C - Aurignacien de Transition

- Datation C14 $\approx 40000$ ans (Cabrera Valdès et al., 1996).

- Richesse pollinique assez élevée - 18 taxons.

(A noter que la grotte est devenue abri après le recul du porche). $35 \%$.

- AP a légèrement régressé par rapport au niveau 20 ; il est voisin de

- Le Pin domine les autres arbres, tempérés thermophiles et méditerranéens qui se partagent le reste du couvert forestier.

- Les xérophiles et/ou steppiques se sont étendues ( $\approx 55 \%$ ).

- Asteraceae et Poaceae sont stables.

- Le taux des Filicales est de $20 \%$.

Ce niveau aurignacien très ancien a dû se déposer sous un climat sensiblement identique à celui qui a accompagné le dépôt moustérien sous-jacent, c'est-à-dire un climat trempéré-froid assez sec appartenant de toute évidence à un épisode du stade isotopique 3. 
6 - Niveau 18B - Aurignacien de Transition

- Datation $C 14 \approx 38500$ ans (Cabrera Valdès et al., 1996).

- Richesse pollinique élevée - 20 taxons.

- AP a encore régressé par rapport au niveau $18 \mathrm{C}$; il n'atteint pas $25 \%$.

- Le Pin s'est effacé ; les taxons arborés tempérés et thermophiles supplantent les méditerranéens.

- Le reste du paysage est largement occupé par les xérophiles et/ou steppiques qui atteignent presque $60 \%$.

- Les Asteraceae ont progressé devant les Poaceae en baisse.

- Le taux des Filicales est au plus bas $(\approx 10 \%)$.

Le climat ayant présidé à ce dépôt aurignacien plus jeune que le précédent, devenu plus froid et plus sec, appartient à un épisode froid du stade isotopique 3.

La sédimentologie caractérise «un climat peu froid à la base, passant à des conditions de température un peu plus rigoureuse au sommet» (Hoyos Gomez, in : Cabrera Valdès et al., 1993).

L'étude anthracologique des niveaux $18 \mathrm{C}$ et $18 \mathrm{~B}$ a permis d'individualiser principalement le Pin et le Bouleau, également présents dans les deux analyses polliniques, accompagnés par des Sorbiers dont l'Alisier (Sorbus aria) et une Légumineuse (Uzquiano y Cabrera, 1999).

Dans la même région cantabrique, nous retrouvons les épisodes froids contemporains des niveaux de l'Aurignacien I et de l'Aurignacien II dans le diagramme pollinique de la Cueva Morin (Leroi-Gourhan, 1971), des niveaux de l'Aurignacien moyen, supérieur et final, dans celui de la grotte d'Isturitz (Leroi-Gourhan, 1960) et des niveaux de l'Aurignacien évolué et tardif dans celui de la grotte du Pendo (Leroi-Gourhan, 1980).

Seule la base du diagramme de la Cueva Morin enregistre une légère amélioration climatique qui peut avoir valeur d'interstade au niveau de l'Aurignacien «O» (fig. 3).

Nous savons que le passage Moustérien - Aurignacien «archaïque» ou "O $\mathrm{\text { }}$ est caractérisé par une période d'instabilité climatique (Leroi-Gourhan et Renault-Miskovsky, 1977) ; ce sont les interstades d'Hengelo et des Cottés également retrouvés, par exemple, dans le sud-est de la France à la Laoûza, Gard (Renault-Miskovsky, 1981). Mais ces différentes données sont difficiles à corréler avec les résultats isolés obtenus au Castillo, surtout pour une date d'Aurignacien de Transition si ancienne. 


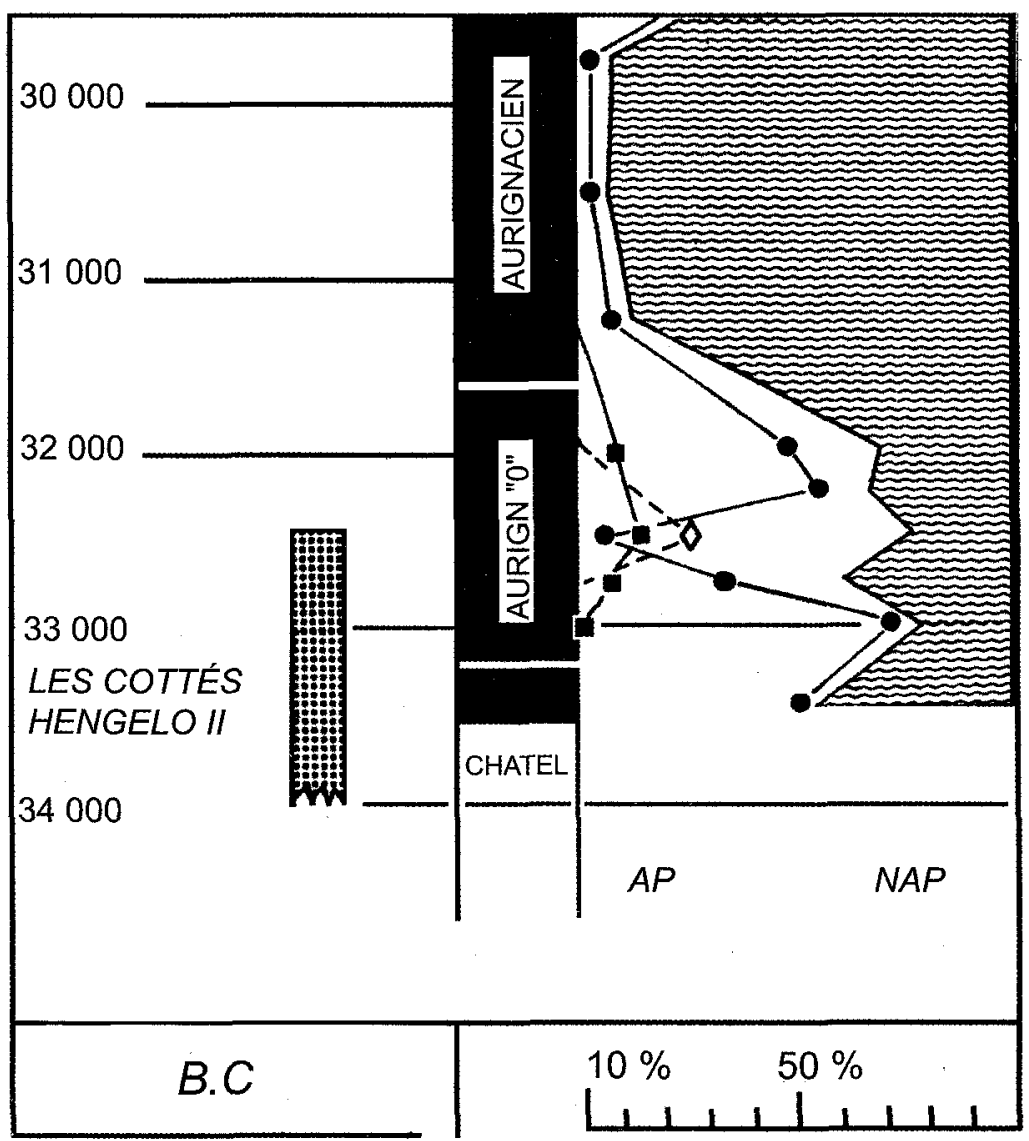

Fig. 3. La Cueva Morín: diagramme pollinique synthétique simplifié des niveaux aurignaciens (Leroi-Gourhan, 1971, in: Leroi-Gourhan et Renault-Miskovsky, 1977).

L'étude des faunes dans les deux sites de la Cueva Morin et du Pendo, permet d'appréhender des variations saisonnières à la limite Paléolithique moyen - Paléolithique supérieur, à rapprocher des résultats obtenus dans la grotte du Castillo (Pike-Tay et al., 1999).

\section{Conclusions à l'étude pollinique}

Cette étude qui porte sur des pluies polliniques fossiles séparées chacune dans le temps par des milliers d'années, enregistre cependant 
la représentation d'une végétation relativement constante dans sa composition par regroupements écologiques dont le Pin a été isolé ; c'est le balancement des valeurs, par niveau, des taxons arborés tempérés et thermophiles, des taxons méditerranéens, des ligneux et herbacées xérophiles et/ou steppiques, des herbacées ubiquistes et dans une moindre mesure des filicales accompagnant semble t-il les sous-bois, qui permet d'attribuer une caractéristique climatique à chaque période culturelle envisagée.

II semble que ce soit le niveau 24 - Acheuléen qui ait enregistré le taux le plus important de pollens d'arbres, tempérés et thermophiles associés aux taxons méditerranéens.

Le niveau suivant, 22 - Moustérien Bêta, est aussi forestier, mais c'est le Pin qui supplante les autres arbres.

Dans les quatre niveaux suivants, niveaux 21 et 20 - Moustérien entre Bêta et Alpha et Alpha et 18C, 18B - Aurignacien de Transition, le paysage est beaucoup plus découvert ; il est très découvert dans le niveau 21 où l'espace est envahie par les ligneux et herbacées xérophiles et/ou steppiques.

Par contre les niveaux 20 - Moustérien Alpha et 18C - Aurignacien de Transition enregistrent une reconquête partielle et temporaire du paysage par le milieu forestier ; il semblerait plausible de voir là une oscillation climatique à valeur d'interstade, au niveau de cette charnière culturelle qui pourrait donc être aussi climatique.

Dans le niveau suivant, 18B - Aurignacien de Transition, la steppe s'étend de nouveau conformément à tous les résultats paléobotaniques recueillis pour l'Aurignacien en Europe ; si le niveau 18B est encore de l'Aurignacien, il y a naturellement contradiction.

Au terme de ces analyses préliminaires, deux remarques s'imposent:

La représentation constante des taxons arborés tempérés et thermophiles associés aux taxons méditerranéens, même au niveau 21, le plus froid et le plus sec, laisse supposer que la grotte du Castillo s'ouvre sur une pente particulièrement abritée des intempéries qui a servi de refuge aux essences végétales et certainement aux espèces animales.

La diversité des taxons regroupés selon leurs exigences écologiques, témoignerait par ailleurs d'une mosaïque de végétations réparties des plus hauts sommets au littoral, au sein des différentes niches écologiques qui leur sont offertes ; cette dernière hypothèse est à retenir pour la dispersion des faunes. 


\section{ETUDE ARCHÉOZOOLOGIQUE}

\section{Matériel d'étude}

Avant d'aborder cette étude, il faut signaler qu'elle porte uniquement sur les grands mammifères des niveaux $20,18 \mathrm{C}$ et $18 \mathrm{~B}$. Les rongeurs, les lagomorphes et les oiseaux ont été classés avec la microfaune, ce qui empêche l'application d'autres méthodes de reconstitutions paléoenvironnementales.

Le matériel osseux considéré provient des nouvelles fouilles menées depuis 1980. C'est un matériel abondant et homogène qui, dans tous les niveaux, représente des populations animales constituées essentiellement d'herbivores $(97,96 \%$ dans le niveau $20,96,77 \%$ dans le niveau $18 \mathrm{C}$ et $96,66 \%$ dans le niveau 18B). Les carnivores sont également représentés mais par de très faibles pourcentages $(2,04 \%$ dans la unite $20,3,22 \%$ dans le niveau $18 \mathrm{C}$ et $3,33 \%$ dans le niveau $18 \mathrm{~B}$ ).

Contrairement à ce que nous avions observé lors de l'étude archéozoologique du matériel osseux provenant des anciennes fouilles de l'abbé H. Breuil et H. Obermaier (Dari, 1998), nous avons constaté un taux de fracturation d'origine anthropique très élevé. L'analyse taphonomique a mis en évidence que $27 \%$ des os (déterminés et indéterminés) du niveau 20 et $29 \%$ de ceux provenant des deux niveaux $18 \mathrm{C}$ et $18 \mathrm{~B}$ présentent des marques d'origine anthropique (impacts de percussion, stries et traces de calcination).

Les dents sont abondantes et présentent toutes un très bon état de conservation ; elles nous ont permis d'estimer le Nombre Minimal d'Individus (NMI) des différentes espèces dans chaque niveau archéologique. Afin de réduire l'effet de la forte fracturation d'origine anthropique, les proportions relatives des différents groupes sont estimées à partir de ce NMI.

\section{Paléoécologie}

La répartition des espèces déterminées dans la unite 20 , et les niveaux $18 \mathrm{C}$ et $18 \mathrm{~B}$ est donnée dans le tableau I.

Seule, la méthode des associations des grands mammifères a donc pu être réalisée, celle-ci prenant en compte les exigences écologiques des différents taxons. Les espèces déterminées sont rangées en quatre groupes écologiques (Tab. II). 
Tabla I. Dénombrement des espèces del'unite 20 (Moustérien), et les niveaux $18 \mathrm{C}$ et $18 \mathrm{~B}$ (Aurignacien)

\begin{tabular}{lcccccc}
\hline & \multicolumn{3}{c}{ Moustérien } & \multicolumn{3}{c}{ Aurignacien archaïque } \\
\hline & \multicolumn{2}{c}{ Unite 20} & \multicolumn{2}{c}{ Niveau } & 18C & \multicolumn{2}{c}{ Niveau 18B } \\
\hline & $N R$ & NMIC & NR & NMIC & NR & NM/C \\
\hline cerf & 1572 & 34 & 4981 & 86 & 5882 & 89 \\
chevreuil & 34 & 4 & 39 & 7 & 95 & 10 \\
sanglier & 0 & 0 & 0 & 0 & 5 & 3 \\
bovinés (Bos/Bison) & 544 & 21 & 190 & 6 & 152 & 5 \\
cheval & 347 & 16 & 19 & 3 & 22 & 3 \\
rhinocéros des prairies & 54 & 12 & 11 & 3 & 13 & 3 \\
bouquetin & 2 & 2 & 6 & 2 & 6 & 2 \\
chamois & 28 & 7 & 155 & 13 & 458 & 29 \\
mammouth & 0 & 0 & 0 & 0 & 1 & 1 \\
\hline Total herbivores & 2581 & 96 & 5401 & 120 & 6634 & 145 \\
\hline loup & 0 & 0 & 4 & 1 & 3 & 1 \\
lion des cavernes & 0 & 0 & 1 & 1 & 0 & 0 \\
panthère & 0 & 0 & 3 & 1 & 0 & 0 \\
hyène & 0 & 0 & 0 & 0 & 3 & 1 \\
ours des cavernes & 4 & 1 & & 0 & 0 & 0 \\
ours brun & 1 & 1 & 1 & 1 & 17 & 3 \\
\hline Total carnivores & 5 & 2 & 9 & 4 & 23 & 5 \\
\hline Total & 2586 & 98 & 5410 & 124 & 6657 & 150 \\
\hline
\end{tabular}

Nous rappelons que cette méthode se heurte à certaines limites d'applications, ce qui nécessite l'intégration des résultats dans le cadre d'une étude interdisciplinaire. En effet, une première question se pose de savoir si les espèces fossiles vivaient vraiment dans les mêmes biotopes que leurs congénères actuels? Nous ne pouvons pas en être certain; par exemple le renne actuel ne vit pas sous la même latitude que celui du Paléolithique; il est donc difficile d'envisager une même nourriture pour l'un comme pour l'autre.

Le deuxième problème, concerne l'origine des assemblages osseux (carnivores ou hommes préhistoriques). Le spectre faunique ainsi établi est souvent non représentatif de la réaiité. Dans ce cas une analyse taphonomique est indispensable avant d'aborder toute interprétation paléoécologique.

La plupart des grands mammifères n'enregistrent pas les fluctuations climatiques courtes ou de faibles amplitudes, ce qui représente une troisième limite. 
Tabla II. Répartition des espèces d'El Castillo selon les groupes écologiques

\begin{tabular}{cccc}
\hline Groupe forestier & $\begin{array}{c}\text { Groupe d'espace } \\
\text { ouvert }\end{array}$ & Groupe ubiquiste & Groupe de montagne \\
\hline $\begin{array}{c}\text { cerf, chamois, } \\
\text { sanglier et } \\
\text { panthère }\end{array}$ & $\begin{array}{c}\text { cheval, bovinés, } \\
\text { rhinocéros }\end{array}$ & $\begin{array}{c}\text { loup, lion des } \\
\text { cavernes et hyéne }\end{array}$ & bouquetin, chamois \\
\hline
\end{tabular}

Enfin la nourriture des herbivores peut également intervenir dans la complexité des interprétations paléoécologiques des sites. En effet, il suffit que des végétaux inconsommables par des animaux envahissent les alentours du site pour que ces derniers partent loin à la recherche de leur nourriture préférée. Nous pensons, ici, au gisement de la Riera dans les Cantabres où le départ et le retour des chevaux et des bovinés étaient liés à l'abondance ou non des bruyères à proximité du site (Straus, 1986 in Leroi-Gourhan Arl., 1989).

Les spectres fauniques établis à El Castillo sont relativement diversifiés (fig. 4), en accord avec ce qui a été observé dans d'autres sites contemporains dans les Cantabres. La présence du mammouth parmi les espèces déterminées dans le niveau 18B est attestée par un seul fragment d'ivoire d'une dizaine de centimètres de longueur. Cette pièce unique qui représente une matière première importante a vraisemblablement fait l'objet d'un échange avec des Aurignaciens d'autres sites et a été introduite dans la grotte. C'est en effet la seule pièce en ivoire découverte dans l'ensemble du site El Castillo.

\section{Résultats concernant l'unite 20 - Moustérien}

D'après les diagrammes des associations des grands mammifères (Fig. 5), on constate que dans l'unite 20, trois groupes écologiques seulement sont représentés, le groupe des ubiquistes étant absent. Le groupe du milieu ouvert est dominant (50\%); il est représenté par des espèces de grandes tailles qui s'adaptent bien à un climat froid et relativement sec. Le groupe du milieu forestier $(41 \%)$, est essentiellement le cerf européen (Cervus elaphus) et dans une moindre mesure le chevreuil (Capreolus Capreolus). Ces cervidés, en particulier le chevreuil, exigent des forêts à feuillus pour se nourrir et s'abriter des prédateurs. Ils préfèrent aussi brouter, en petites hardes, de l'herbe tendre 
Unite 20 - Moustérien
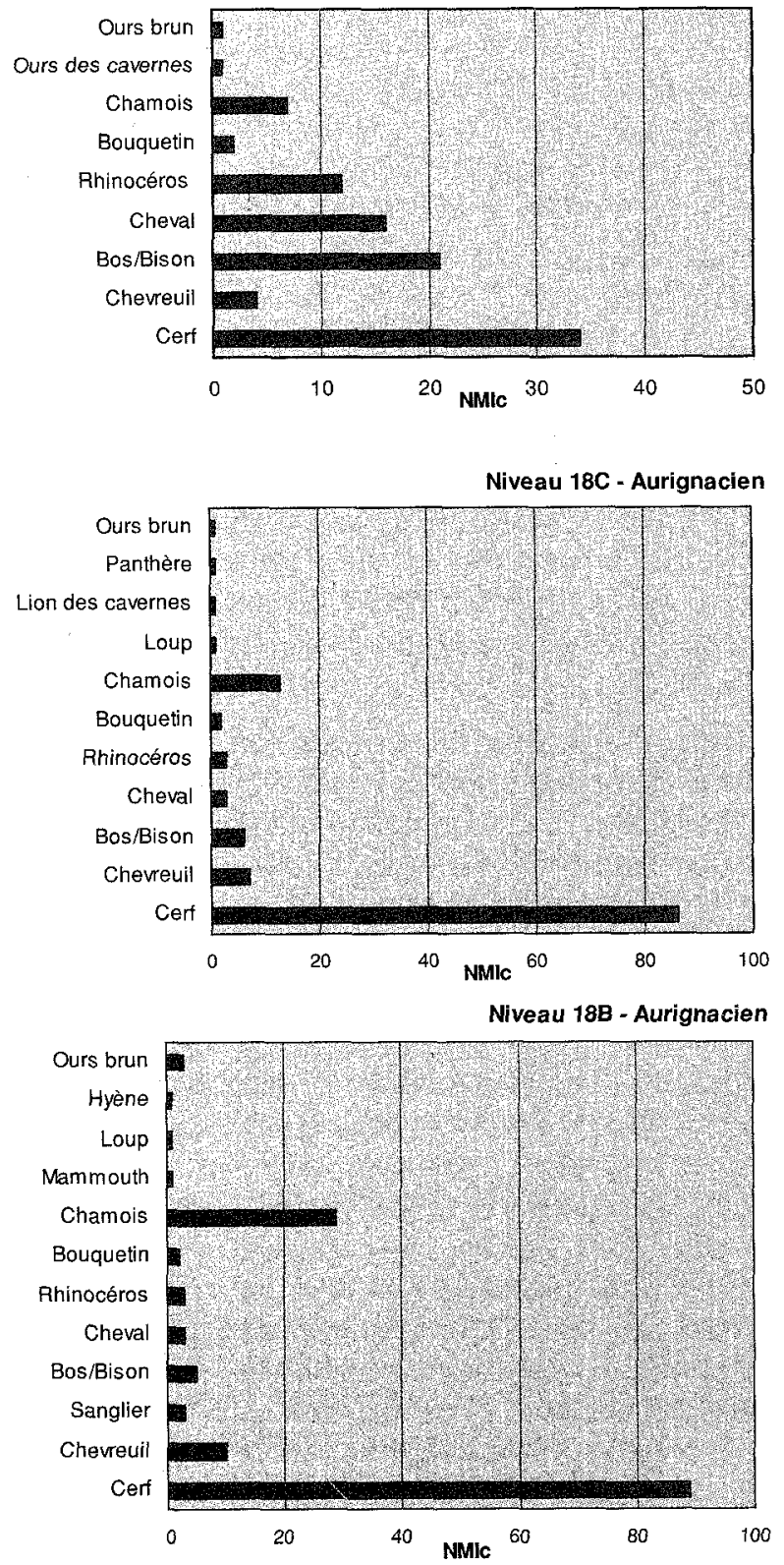

Fig. 4. Dénombrement en NMic des espèces déterminées dans les niveaux (Moustérien), $18 \mathrm{C}$ et $18 \mathrm{~B}$ (Aurignacien). 

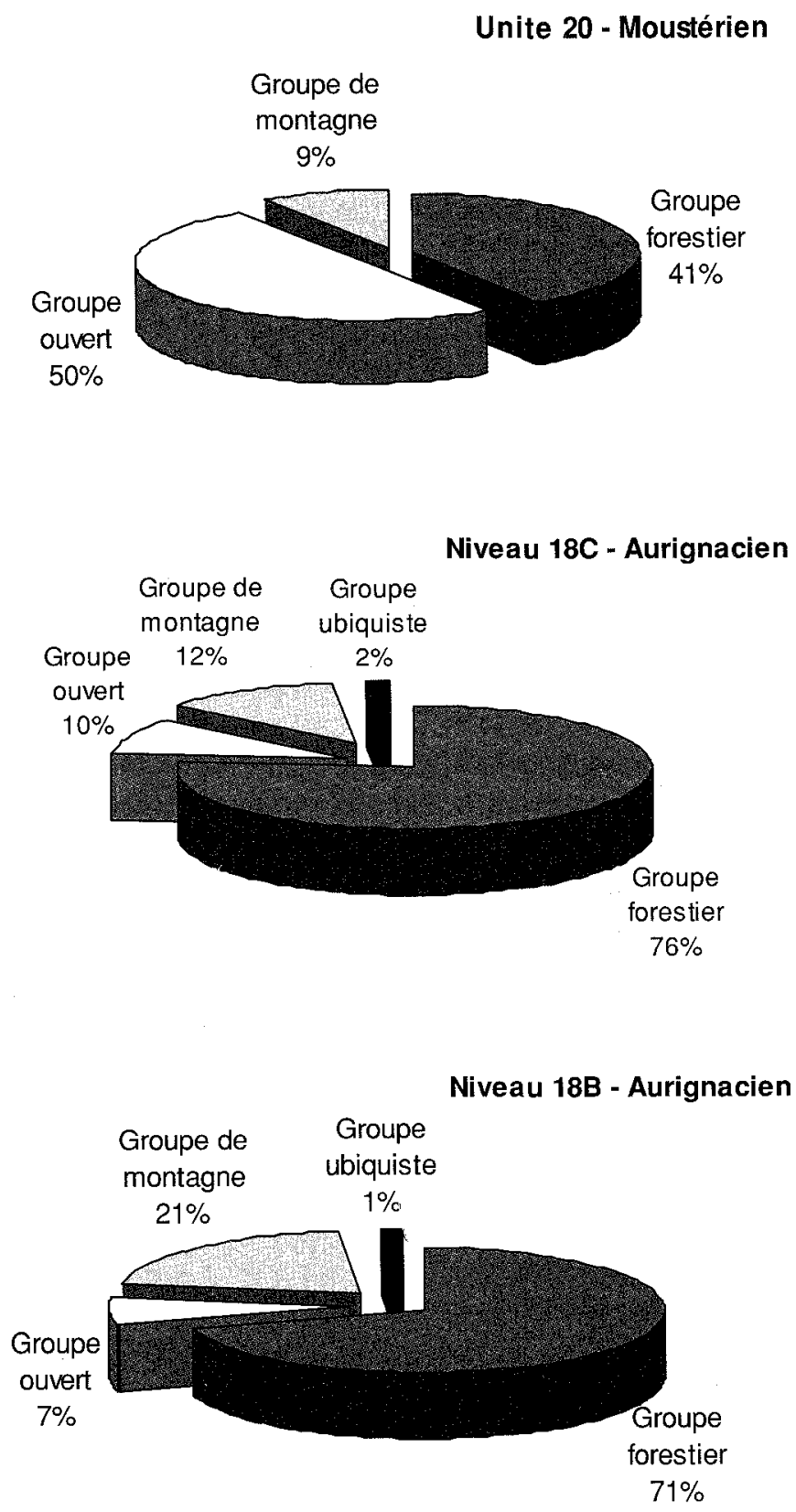

Fig. 5. Pourcentage relatif des 4 groupes écologiques définis à partir des NMIC dans l'unite 20 (Moustérien), et les niveaux $18 C$ et $18 B$ (Aurignacien). 
dans les prairies avoisinantes. Le climat peut être moyennement froid à froid, mais constamment humide (Delpech, 1983). Enfin le groupe de montagne (au moins sept chamois et deux bouquetins) représente $9 \%$; ces bovidés habitent dans les hautes altitudes escarpées et supportent bien le froid tout en évitant les glaciers. Quand leurs territoires sont envahis par la neige, les chamois descendent pour s'abriter dans les forêts ce qui explique alors leur abondance par rapport aux bouquetins qui n'y entrent pas.

On peut imaginer que pendant la mise en place de l'unite 20 , un couvert végétal, relativement important s'est installé, à l'abri du froid, dans la vallée de la rivière «EI Pas» et sur les montagnes d'altitudes moyennes dont le mont El Castillo. Les plateaux qui s'étendent sur plusieurs kilomètres depuis la côte jusqu'aux pieds des montagnes cantabriques, offrent de véritables pâturages pour les chevaux, les bovinés et les rhinocéros des prairies. Le climat est tempéré et relativement sec.

2. Résultats concernant les niveaux $18 \mathrm{C}$ et $18 \mathrm{~B}$ - Aurignacien de Transition

Le passage au Paléolithique supérieur est marqué par le développement frappant du groupe forestier représenté essentiellement par le cerf élaphe ( $76,6 \%$ dans le niveau $18 \mathrm{C}$ et $71 \%$ dans le niveau $18 \mathrm{~B})$. Le chevreuil est plus abondant que dans le niveau précédent. On note également la régression progressive des espèces d'espaces ouverts (bovinés, équidés et rhinocéros de prairies).

Le groupe de montagne représenté essentiellement par le chamois (deux bouquetins seulement ont été déterminés dans chacun des deux niveaux $18 \mathrm{C}$ et $18 \mathrm{~B}$ ) se maintient et devient relativement abondant notamment dans le niveau 18B. Cette importance du chamois pourrait être expliquée par des enneigements fréquents en altitude.

Notons l'apparition sporadique du groupe ubiquiste (loup hyène et lion) dans ces deux niveaux aurignaciens de Transition. Par précaution, nous l'avons écarté de nos interprétations pour deux raisons : d'une part, il regroupe des espèces dépourvues de signification climatique précise du fait qu'elles s'adaptent facilement à plusieurs types de climats, d'autre part, il n'est représenté que par un nombre réduit d'individus (2\% dans le niveau $18 \mathrm{C}$ et $1 \%$ dans le niveau $18 \mathrm{~B}$ ).

Ces variations des pourcentages des groupes écologiques, notamment, entre l'unite 20 et le niveau $18 \mathrm{C}$ ne reflètent pas a fortiori d'importantes variations climatiques et environnementales. Le cerf qui était abondant, sem- 
ble-il, et fréquemment chassé par les Moustériens est très bien représenté pendant l'Aurignacien. Les chasseurs ont peut être préféré cette source alimentaire principale plutôt que d'aller parcourir de larges espaces découverts situés plus au nord à une dizaine de kilomètres pour s'attaquer à des gibiers de grandes tailles, souvent redoutables, et dépenser ainsi un maximum d'énergie. Plusieurs autres hypothèses peuvent être envisagées.

- La chasse s'est orientée, pendant l'Aurignacien, vers les cerfs élaphes mâles dans un but culturel lié au travail des bois. L'idée nous est venue lorsque nous avons examiné les dents; celles-ci présentent, pour la plupart, des dimensions élevées qui sont proches de celles des dents d'individus mâles. L'étude d'un éventuel dimorphisme sexuel à partir des dents est en cours; les résultats permettront de confirmer ou d'infirmer cette hypothèse.

- Les groupes humains occupant le site pendant l'Aurignacien étaient peut-être numériquement plus importants que durant le Moustérien ; les occupations semblent en effet plus nombreuses et plus rapprochées dans les niveaux $18 \mathrm{C}$ et $18 \mathrm{~B}$, notamment dans le niveau $18 \mathrm{C}$ où l'on a découvert plusieurs foyers. Le nombre plus important de bêtes à abattre aurait donc du dans ce cas répondre aux besoins alimentaires des groupes.

- L'inégalité des surfaces fouillées, $3 \mathrm{~m}^{2}$ pour l'unite 20 par rapport à $12 \mathrm{~m}^{2}$ environ pour les niveaux $18 \mathrm{C}$ et $18 \mathrm{~B}$, est également à prendre considération.

En tenant en compte de ces données et de ces hypothèses, on peut imaginer, pendant la mise en place du niveau $18 \mathrm{C}$, un paysage en mosaïques (forestier et ouvert), relativement semblable à celui du niveau précédent, et établi sous un climat tempéré et humide.

Autres points intéressants, l'apparition du sanglier (Sus scrofa) (trois individus minimum) dans le niveau 18B. Cet animal habite essentiellement des forêts de feuillus à proximité de lacs et de marécages et supporte des écarts de températures considérables (Faure et Guérin, 1984). Sa présence dans un gisement archéologique traduit l'installation proche d'un paysage arboré relativement dense. Par contre, l'abondance des gibiers de tailles moyennes, cerf, chevreuil et occasionnellement quand il fait très froid de chamois, s'est maintenue dans le niveau 18B. Les grands herbivores de prairies, étant loin de la grotte, à une dizaine de kilomètres, ne sont qu'occasionnellement abattus et dépecés sur le lieu même de la chasse, ce qui expliquerait la rareté de leurs restes dans les deux niveaux $18 \mathrm{C}$ et $18 \mathrm{~B}$. 


\section{Conclusions à l'étude archéozoologique}

Le fait que les spectres fauniques soient plus ou moins semblables tout le long des séquences stratigraphiques des gisements archéologiques du nord de l'Espagne (Pays basque, Cantabres et Asturies) est un phénomène habituel. En effet, cette région est considérée comme une zone de refuge pour de nombreuses espèces nordiques lors de leurs migrations saisonnières (Altuna, 1971, 1972, 1989, 1990 ; Altuna et Mariezkurrena, 1984, 1985 ; Dari, 1999 ; Klein et Cruz-Uribe, 1994).

- Trois causes géologiques et climatiques semblent être à l'origine de cette grande diversité spécifique de la faune des grands mammifères dans la région.

- La topographie est particulièrement variée. En effet, à quelques kilomètres seulement de la côte (fig. 1), le paysage change énormément : d'abord les plateaux qui s'étendent sur une dizaine de kilomètres s'élèvent à de moyennes altitudes dont le mont El Castillo, enfin les montagnes peuvent atteindre des altitudes considérables (jusqu'à $1000 \mathrm{~m}$ dans le pays basque, 1500 dans les Cantabres et jusqu'à $2000 \mathrm{~m}$ au niveau des Asturies).

- La zone est parcourue, transversalement, par de nombreuses rivières encaissées dans des vallées très profondes qui, parfois, représentent des barrières géographiques infranchissables pour les animaux de grandes tailles comme les rhinocéros et les bovinés. De ce fait, des territoires plutôt restreints offraient aux chasseurs une capture stratégique et facile. Ces vallées d'allure méandriformes étaient de véritables obstacles aux vents froids et secs venants du nord.

- Les chaînes montagneuses, situées à une vingtaine de kilomètre seulement de la côte, arrêtent toutes les fluctuations maritimes pluvieuses venant du large qui affectent alors directement les versants nord de ces montagnes.

Le climat local semble être constamment doux et humide ce qui aurait favorisé l'installation d'un couvert arboré quasi-permanent et varié selon les altitudes. Des biotopes différents mais spatialement relativement rapprochés les uns des autres se seraient constitués.

J. Altuna $(1989,1990)$, en s'appuyant sur des exemples précis, avait d'ailleurs déjà fait remarquer que c'était la situation géographique qui intervenait directement sur la composition spécifique des spectres fauniques issus des gisements archéologiques magdaléniens de cette région. II a subdivisé ces sites magdaléniens en deux principales catégories: 
Quand le site est installé dans une zone à relief peu abrupt comme c'est la cas pour les gisements Tito Bustillo - niveau 1 (Altuna, 1976), El Pendo - niveau 2 (Fuentes, 1980), Morin - niveau 2 (Altuna, 1971) et Ekain - niveau VII (Altuna et Mariezkurrena, 1984), la chasse est orientée vers le cerf. La liste faunique est constituée, jusqu'à $80 \%$, par cet animal qui semble représenter la source alimentaire fondamentale des groupes humains. Le reste de la faune est partagé entre d'autres ongulés notamment le bouquetin, les bovinés, le cheval, et le chamois.

Quand le gisement se trouve en pleine montagne dans une zone rocheuse et escarpée comme c'est la cas pour les trois sites cantabriques Rascaño - niveau 3 (Altuna, 1981), Ermittia - niveau 3 (Altuna, 1972) et Erralla - niveau V (Altuna et Mariezkurrena, 1985), c'est plutôt le bouquetin qui domine à $80 \%$ par rapport aux autres ongulés.

En ce qui concerne le gisement El Castillo, nous rappelons que dans tous les niveaux archéologiques, de l'Acheuléen à l'Azilien, le bouquetin n'est représenté que par quelques rares restes osseux (Dari, 1999). Par sa situation géographique facilement accessible, nous introduisons donc le site El Castillo dans la première catégorie de J. Altuna.

\section{CONCLUSION GÉNÉRALE}

C'est la comparaison entre les résultats de la palynologie et ceux de l'archéozoologie qui peut nous servir de réflexion sur l'interprétation des données et ses limites; elle ne concerne que les niveaux qui se sont prêtés à une étude faunistique, c'est à dire l'unite 20 (Moustérien), et les niveaux $18 \mathrm{C}$ et 18B (Aurignacien de Transition).

\section{Unite 20 - Moustérien Alpha (datation $C 14 \approx 43-45000$ ans)}

- La palynologie documente un couvert arboré qui occupe à peu près $40 \%$ du paysage partagé entre les Pins, les taxons tempérés et thermophiles et quelques genres méditerranéens et qui se serait installé sous un climat tempéré-froid relativement $\mathrm{sec}$ propice à l'ouverture de la forêt et au développement des espèces steppiques.

- La faune apparaît essentiellement constituée d'herbivores $(97,96$ $\%)$, dont de nombreux herbivores de prairie (50\%), le groupe des espèces forestières représentant $41 \%$ et le groupe de montagne étant restreint à $9 \%$. 
Niveau 18C - Aurignacien de Transition (datation C14 $\oplus 40000$ ans)

- La palynologie suggère une réduction du couvert arboré dominé par le Pin et une extension des espaces ouverts xérophiles ou steppique, développés à la faveur d'un climat tempéré-froid et sec proche du climat évoqué précédemment.

- Les éléments de la faune sont principalement des herbivores $(96,77$ $\%)$. Le groupe occupant les espaces ouverts est de $10 \%$, le groupe des montagnards est encore assez bas (12\%), par contre le groupe forestier atteint $76 \%$ avec de nombreux cervidés (principalement des cerfs).

Niveau $18 B$ - Aurignacien de Transition (datation C14 $\approx 38500$ ans)

- La palynologie confirme l'extension des espaces ouverts occupés par des herbacées xériques ou steppiques, dont les Asteraceae, inféodés à un climat plus froid et plus sec que celui ayant présidé au dépôt du niveau $18 \mathrm{C}$.

- La faune est toujours dominée par les herbivores $(96,66 \%)$; la répartition par groupe dans différents niveaux est la suivante : ouvert $7 \%$, de montagne $21 \%$, ubiquiste $1 \%$ et forestier $71 \%$ avec $84,76 \%$ de cerfs (auxquels il faut ajouter le chevreuil, le sanglier et l'ours brun).

Nous avons déjà souligné l'importance de la situation de la grotte El Castillo au carrefour de plusieurs niches écologiques, entre la mer et la haute montagne, pour expliquer d'une part, la variété des associations végétales en mosaïques, et d'autre part la diversité des biotopes capables d'accueillir les différents groupes d'herbivores suivant leurs exigences.

Néanmoins, il faut considérer que les résultats de l'archéozoologie et de la palynologie sont apparemment en contradiction aux niveaux $18 \mathrm{C}$ et 18B :

- La palynologie fait état d'un climat tempéré - froid ou froid et sec responsable de la mise en place d'espaces ouverts plus ou moins steppiques, alors que l'inventaire des faunes tendrait à mettre en évidence l'implantation d'un couvert forestier abritant d'importantes hardes de cervidés (chamois et cerfs), avec des sangliers ; l'abondance du chamois dans le niveau $18 \mathrm{C}$ pourrait par ailleurs témoigner d'une pluviométrie élevée en altitude qui aurait contraint l'animal à descendre plus bas dans la forêt pour pouvoir s'abriter. 
Nous devons cependant objectivement considérer que l'origine de l'assemblage osseux est incontestablement anthropique ; de ce fait, les interprétations paléoécologiques à partir de la grande faune sont à prendre avec beaucoup de précaution. Les populations animales déterminées ne reflètent d'une façon certaine que le choix des chasseurs. Ainsi ce choix, qu'il soit alimentaire ou culturel peut dissimuler la réelle dispersion de la faune régionale. Dans cet ordre d'idée, il est possible d'admettre que l'exceptionnelle représentation du cerf à l'Aurignacien peut représenter le produit d'une chasse sélective pour l'utilisation des bois, en relation avec le début d'une évolution culturelle propre au Paléolithique supérieur par rapport au Moustérien.

II convient également de prendre en compte les derniers résultats concernant l'apport des isotopes stables du carbone dans les os des anjmaux qui permet d'illustrer les rapports entre l'animal et son alimentation végétale ; ainsi, $\mathrm{H}$. Bocherens (2001) a démontré que la gamme en ${ }^{13} \mathrm{C}$ du cerf (Cervus elaphus) est très étendue, d'un milieu ouvert à un milieu fermé.

La climatologie et l'environnement végétal des derniers Néandertaliens et des premiers hommes modernes, durant l'Aurignacien sont par ailleurs maintenant bien documentés nous l'avons vu pour le nord de l'Espagne à l'échelle régionale (Cueva Morin, grotte d'Isturitz, grotte du Pendo), dans le reste de la péninsule ibérique à l'Arbreda en Catalogne (Burjachs et Renault-Miskovsky, 1992), aux Malladetes, Valence (Dupré Ollivier, 1980), à la Cova Beneito, Alicante (Carrion, 1992) et dans le reste de l'Europe par exemple à la grotte Tournal, Aude en France (Farbos-Texier, 1982), à l'Abri Mochi en ligurie italienne (Renault-Miskovsky, 1972) et à Geissenklösterle dans le sud de l'Allemagne (Wille, 1978).

Nous nous permettons néanmoins d'émettre quelques souhaits pour l'avenir: pouvoir disposer de nouvelles datations absolues pour cet Aurignacien particulièrement ancien et des résultats de l'étude parallèle des petits mammifères (rongeurs et lagomorphes) et des oiseaux, qui offriraient la possibilité d'appliquer d'autres méthodes; celles des cénogrammes et des diagrammes écologiques apporteraient des compléments d'informations paléoenvironnementales.

\section{Remerciements}

Nous tenons à remercier le Professeur Denis VIALOU, responsable du «GDR 1945 », pour son soutien, en acceptant de financer certains dé- 
placements à Madrid, le traitement chimique des échantillons palynologiques en laboratoire et l'informatisation des résultats.

Nous exprimons aussi notre gratitude aux Professeurs Victoria CABRERA VALDES et Federico BERNALDO DE QUIROS pour leurs conseils et pour avoir bien voulu mettre à notre disposition, six échantillons sédimentaires appartenant à des niveaux culturels différents, afin de tenter une étude pollinique préliminaire.

Abdellah DARI est heureux de remercier son Professeur, Marylène PATOU-MATHIS, pour son encadrement et ses fructueux conseils

Enfin, Josette RENAULT-MISKOVSKY remercie sincèrement David KANIEWSKI qui a réalisé l'extraction du matériel sporo-pollinique en laboratoire et l'enregistrement informatique des données numériques.

\section{BIBLIOGRAPHIE}

ALTUNA J. (1971): Los mamíferos del yacimiento prehistórico de Morín (Santander). In : González Echegaray J. y Freeman L.G. (eds). Cueva Morín. Excavaciones 1966-68, Publicaciones del Patronato Cuevas Prehistóricas de Santander 6, Santander, págs. 367-398.

AltunA, J. (1972): Fauna de mamíferos de los yacimientos prehistóricos de Guipúzcoa. Munibe 24, San Sebastian, págs. 1-4.

ALtUNA, J. (1976) Los mamíferos del yacimiento prehistórico de Tito Bustillo (Asturias). In : Moure J. A. y Cano M. Excavaciones en la cueva de Tito Bustillo (Asturias). Instituto de estudios asturianos, Oviedo, págs. 149-194.

ALtuna, J. (1981): Restos óseos del yacimiento prehistórico del Rascaño. In : González Echegaray J. y Barandiarán I. El Paleolítico superior de la cueva del Rascaño (Santander). Centro de investigación y Museo de Altamira. Monografías 3, Santander, págs. 221-269.

ALtUNA J. (1989): Subsistance d'origine animale pendant le Moustérien dans la région cantabrique. In : L'Homme de Néandertal, Eraul, vol. 6. La subsistance, Liège, págs. 31-43.

ALTUNA J. (1990): Caza y alimentación procedente de Macromamíferos durante el Paleolítico de Amalda. In : Altuna J., Baldeón A. y Mariezkurrena, K. La cueva de Amalda (País Vacco). Ocupaciones paleolíticas y postpaleolíticas. Eusko Ikaskuntza Serie B 1, San Sebastian, págs. 149-192.

ALtUNA J. et MARIEZKURRENA K. (1984): Bases de subsistencia de origen animal en el yacimiento de Ekain, In : Altuna J. y Merino J.M. El yacimiento prehistórico de la cueva de Ekain (Deva, Guipúzcoa). Eusko Ikaskuntza Serie B 1, San Sebastian, págs. 211-280.

ALtUNA J. et MARIEZKURRENA K. (1985): Bases de subsistencia de los pobladores de Erralla: Macromamíteros. In: ALTUNA J., Baldeón A. y Mariezkurrena, K. Cazadores Magdalenienses en Erralla (Cestona, País Vasco). Munibe (Antropologia, Arqueologia) 37, San Sebastian, págs. 87-117.

BAFFIER, D. (1999): Les derniers Néandertaliens. Le châtelperronien. Histoire de la France de 36000 à -30000 ans. La maison des roches éditeur. 113 pág.

BOCHERENS, H. (2001): Apport des isotopes stables du carbone dans la connaissance de l'exploitation des végétaux par les populations archéologiques. In Comptes rendus de la séance de la Société Préhistorique Française - Toulouse - 19 mai 2001- L'archéobotanique : dernières acquisitions, nouvelles tendances, par M. C. Marinval. Bulletin de la Société Préhistorique Française, octobre-déc. 2001 - Tome 98 - numéro 4, págs. 743-751.

BurJachs, F. et Renault-Miskovsky, J. (1992): Paléoenvironnement et Paléoclimatologie de la Catalogne durant près de 30000 ans (du würmien ancien au début de l'Holocène), d'après la palynologie du site de l'Arbreda (Gérone, Catalogne). Quaternaire, 3 (2), 1992, págs. 75-85. 
Cabrera Valdes, V.; Hoyos Gómez, M.; Bernaldo de Quirós, F. (1993): La transition du Paléolithique moyen au supérieur dans la grotte de «El Castillo» : Caractéristiques paléoclimatiques et situation chronologique. Congrès National des Sociétés historiques et scientifiques 118e, Pau, 1993, págs. 27-40.

Cabrera Valdes, V.; Valladas, H.; Bernaldo de Quiros, F. et Hoyos Gómez M. (1996): La transition Paléolithique moyen - Paléolithique supérieur à El Castillo (Cantabrie) : nouvelles datations par le Carbone 14, C.R. Acad. Sc., Paris, t. 322, série lla, págs. 1093-1098.

CARRION J. S. (1992): A palaeoecological study in the western Mediterranean area. The upper Pleistocene pollen record from cova Beneito (Alicante, Spain) Palaeogeography, Palaeoclimatology, Palaeoecology, 92 (1992), págs. 1-14, Elsevier Science Publishers.

DARI, A. (1999) Les grands mammifères du site pléistocène supérieur de la grotte El Castillo : étude archéozoologique ; données paléontologiques, taphonomiques et paleothnographiques. Espacio, Tiepo y Forma, Rev. fac. Geo e His. (UNED). Serie l, Madrid. págs. 103-127.

DeLPECH, F. (1983): Les faunes du Paléolithique supérieur dans le sud-ouest de la France. Chier du quaternaire $n^{\circ} 6$, CNRS, Paris, 453 págs.

DeLPORTE, H. (1998): Les Aurignaciens premiers hommes modernes. Histoire de la France de 40000 et -25000 ans. La maison des roches éditeur, 125 págs.

Dupré Ollivier, M. (1980): Analisis polinico de sedimentos arqueologicos de la Cueva de las Malladetes (Barx, Valencia), Cu. de geogr., 26, págs. 1-22, Valencia.

Farbos-TeXIER, S. (1982): Etude des flores et des climats de la fin du Moustérien au début du Paléolithique supérieur en Languedoc oriental. Palynologie des sédiments des grottes du Salpêtre de Pompignan (Gard) et Tournal (Aude). Thèse de $3^{\text {ème }}$ cycle. Université de Provence, Marseille.

Faure, M. et Guerin, C. (1984): Sus Strozzii et Sus Scrofa, deux Mammifères artiodactyles, marqueurs des paléoenvironnements. Palaeogeography, Palaeoclimatology, Palaeoecology, 48 , págs. $215-228$.

Feblot-Augustins, J. (1997): La circulation des matières premières au Paléolithique. Eraul, 75 , 2 tomes, 275 págs.

FUENTES C. (1980): ESTUDIO DE LA FAUNA DE EL PENDO. IN : GONZALEZ ECHEGARAY J. (ED.), EL YACIMIENTO DE LA CUEVA DE EL PENDO. EXCAVACIONES 1966-68. BIBLIOTHECA PRAEHISTORICA HISPANA, XVII, págs. 215-238.

KLEIN, R.G. et Cruz-URIRE, K. (1994): The Paleolithic mammalian fauna from the 1910-14 excavations at el Castillo cave (Cantabria). In : LASHERAS (ed.) Homenaje al Dr Joaquin González Echegaray. Museo y Centro de Investigación de Altamira. Monografía 17, Santander, págs. 14t-158.

LeRoI-Gourhan Arl. (1960): Résultats de l'analyse pollinique de la grotte d'Isturitz. Extrait du Bull. de la S.P.F., LVI, 1959, nº 9-10, págs. 619-624.

Lerol-GourHAN, Arl. (1971): Cueva Morin. Excavaciones 1966-1968. VII : Analisis polinico de Cueva Morin. Publicaciones del Patronato de las Cuevas prehistoricas de la provincia de Santander, págs. 359-365.

Lerol-Gourhan, Arl. (1980): El yacimiento de la cueva de "El Pendo" Madrid. Instit esp. de Prehistoria, págs. 265-266.

Leroi-Gourhan, Arl. (1989): Faunes et flores. In : Mohen J.-P. (dir.), Le temps de la Préhistoire, Tome 2. Société Préhistorique Française, Edition Archéologia, Dijon, págs. 54-59.

LEROI-GouRHAN Arl. et RENAULT-MiskovSKY, J. (1977): La palynologie appliquée à l'archéologie (méthodes, limites et résultats) $I n$ : Approche écologique de l'Homme fossile. Supplément au Bulletin de l'AFEQ, $\mathrm{n}{ }^{\circ}$ 47, págs. 35-49, 6 fig.

Martínez MoRino J. (1998): E Modo de vida Neandertal : Una reflexión en torno a la ambigüedad in la interpretación de la subsistencia durante el Paleolítico médio Cantábrico. Thèse de $3^{\text {ème }}$ cycle. Université de Barcelone, 639 págs.

Moure Romanillo, A. (1994): Arte Paleolítico y geografias sociales. Asentamiento, Mobilidad y Agregación en el final del Paleolítico cantábrico. Complutum, n ${ }^{\circ}$ 5. págs. 313-330.

Patou-Mathis, M. (1997): Les grands Mammifères de la couche 5 de Mutzig (Bas-Rhin). La subsistance au Paléolithique moyen en Alsace. Anthropozoologica, $n^{\circ} 25-26$. págs. 363374.

Pike-Tay, A.; Cabrera Valdes, V.; Bernaldo de Quiros, F. (1999): Seasonal variations of the Middle-Upper Paleolithic transition at El Castillo, Cueva Morin and El Pendo (Cantabria, Spain). Journal of Human Evolution (1999), 36, págs. 283-317. 
RenAult-Miskovsky, J. (1972): Contribution à la paléoclimatologie du Midi méditerranéen pendant la dernière glaciation et le postglaciaire, d'après l'étude palynologique du remplissage des grottes et abris sous-roches. Bull. du Musée d'Anthropologie de Monaco, $n^{\circ} 18$, págs. 145-210, 7 fig., 11 tabl.

RENAULT-MISKOVSKY, J. (1981): La Laoûza (Sanilhac-Sagriès, Gard). Analyse pollinique. Etudes quaternaires languedociennes. Mémoire $n^{\circ} \uparrow, 1981$, págs. 31-34.

Rink, W.J.; Schwarcz, H.P., Lee, H.K.; Caerera Valdes, V.; Bernaldo de Quiros, F.; Hovos, M. (1997): ESR dating of Mousterian levels at El Castillo Cave, Cantabria, Spain. Journal of Archaeological Science (1997), 24, págs. 593-600.

UzQUiano, P., y CABRERA V. (1999): Paleoecologia y gestión del combustible en la ocupación del auriñacience arcaico de la cueva de El Castillo (Puente Viesgo, Cantabria). Espacio, Tiempo y Forma, Serie I, Prehistoria y Arqueologia, t. 12, 1999, págs. 129-147.

VALENSI, P. et ABBASSI, M. (1998): Reconstitution de paléoenvironnements quaternaires par l'utilisation de diverses méthodes sur une communauté de Mammifères. Application à la grotte du Lazaret. Quaternaire, vol. 9. n. ${ }^{\circ}$ 4. págs. 291-302.

WILLE, W. (1978): Pollenanalytische Untersuchungen in Jungpalaölithischen $H$ _hlensedimenten des Geinssenklösterles (Södwestdeutschland). Neues Jahrbuch für Geologie und Paläontologie Abhandlungen, Paleoecology, Band 157, Heft 1/2, págs. 261-265. 